\title{
From globular clusters to the disc: the dual life of our Galaxy
}

\author{
Alejandra Recio-Blanco
}

\begin{abstract}
Université Côte d'Azur, Observatoire de la Côte d'Azur, CNRS, Laboratoire Lagrange, France
e-mail: arecio@oca.eu
\end{abstract}

Received 6 April 2018 / Accepted 25 October 2018

\begin{abstract}
The halo and disc globular cluster population can be used as a tracer of the primordial epochs of the Milky Way formation. In this work, literature data of globular clusters ages, chemical abundances, and structural parameters are studied, explicitly focussing on the origin of the known split in the age-metallicity relation (AMR) of globular clusters. When the $\alpha$-element abundances, which are less strongly affected by the internal light-element spread of globular clusters $(\mathrm{Si}, \mathrm{Ca})$, are considered, a very low observational scatter among metal-poor clusters is observed. A plateau at $[\mathrm{SiCa} / \mathrm{Fe}] \sim 0.35 \mathrm{dex}$, with a dispersion of only 0.05 dex (including abundance errors) is observed up to a metallicity of about -0.75 dex. Only a few metal-poor clusters in this metallicity interval present low $[\mathrm{SiCa} / \mathrm{Fe}]$ abundances. Moreover, metal-rich globular clusters show a knee in the $[\alpha / \mathrm{Fe}] \mathrm{vs}$. $[\mathrm{Fe} / \mathrm{H}]$ plane around $[\mathrm{Fe} / \mathrm{H}] \sim-0.75$ dex. As a consequence, if a substantial fraction of galactic globular clusters has an external origin, they have to be mainly formed either in galaxies that are massive enough to ensure high levels of $\alpha$-element abundances even at intermediate metallicity, or in lower mass dwarf galaxies accreted by the Milky Way in their early phases of formation. Finally, clusters in the metal-poor branch of the AMR present an anti-correlation of $[\mathrm{SiCa} / \mathrm{Fe}]$ with the total cluster magnitude, while this is not the case for metal-rich branch clusters. In addition, this lack of faint high- $\alpha$ clusters in the young metal-poor population is in contrast with what is observed for old and more metal-poor clusters, possibly reflecting a higher heterogeneity of formation environments at lower metallicity. Accretion of high-mass satellites, as a major contribution to the current Milky Way globular cluster system both in the metal-poor and the metal-intermediate regime is compatible with the observations.
\end{abstract}

Key words. globular clusters: general - Galaxy: halo - Galaxy: stellar content

\section{Introduction}

In the era of Milky Way surveys, photometric, spectroscopic, and astrometric data for large numbers of stars are rapidly changing our view of the Galaxy. The fingerprints of different galactic evolutionary processes can now be revealed in the chemodynamical characteristics of the stellar populations, with databases increasing every day. The isolated, time-frozen snapshots of separated galactic components today become a moving picture that progressively unveils the Milky Way history. In this context, the comparative analysis of what looked like, in principle, different populations (e.g. the halo and the disc) is crucial to detect the signatures of major evolutionary events that globally affect the Galaxy formation.

Globular clusters (GC) have always been a key population for Galactic archaeology studies. They are witnesses of the ancient times of the Milky Way history, with ages spanning over five gigayears and overlapping the halo and the primordial disc formation. The galactic GC system is therefore at the cross-roads of two key Milky Way stellar populations, and it is precisely this characteristic that this analysis wishes to highlight.

Moreover, GC formation points out the fundamental question of galaxy in situ vs. ex situ, or accretion formation. In simulations, the in situ and/or ex situ origin of a population can be tagged and tracked separately. However, the situation is much more complex in observations. The distinction between GCs that formed in situ and those formed in another galaxy that were subsequently accreted is blurred when detailed physical processes are taken into consideration. For instance, clumpy dissipative collapse, inflowing of cold gas into a turbulent gas disc, mergers of gas-rich galaxies originating in situ formation, etc. are processes that highlight the weakness of a sharp classification into in situ vs. accretion scenarii.

In recent years, several studies have analysed the properties of the Galactic GC age-metallicity relation (AMR). First of all, the increase in age dispersion as a function of the metallicity has been noted (e.g. Buonanno et al. 1998; Rosenberg et al. 1999; VandenBerg 2000; Salaris \& Weiss 2002; De Angeli et al. 2005). Later on, Marín-Franch et al. (2009), Forbes \& Bridges (2010), and Dotter et al. (2011) reported that the AMR exhibited a split, with one sequence of old clusters at all metallicities, and another sequence of intermediate-metallicity clusters. GC accretion has currently been invoked to explain the spread of the AMR.

More recently, Leaman et al. (2013) used the age estimates of VandenBerg et al. (2013) to reveal that the AMR bifurcates at $[\mathrm{Fe} / \mathrm{H}] \sim-1.8 \mathrm{dex}$, with clusters having halo type or disc-like orbits that populate different branches. Leaman et al. (2013) suggested that the metal-rich branch of the AMR consists of clusters that formed in situ in the disc, while the metal-poor clusters could have been formed in relatively low-mass (dwarf) galaxies and later accreted by the Milky Way. More generally, it is currently assumed that metal-rich GCs are mainly an in situ population because the stellar components of massive early-type galaxies and their red GCs share many physical properties (e.g. Pota et al. 2013). In this sense, metal-poor GCs seem to be better candidates for accretion.

Nevertheless, the real observational situation is much more complex. In particular, radial chemical gradients have been detected in several GC systems (e.g. Harris 2009), being 
probably similar for both metal-rich and metal-poor GCs (Forbes et al. 2011). Although these gradients appear to be related with some sort of in situ formation, the invoked scenario should be able to explain them, regardless of which scenario it is. In parallel, many clear signatures of accretion in the halo have been revealed, and the Sagittarius dwarf galaxy and its associated streams and GCs is an outstanding example of it.

From the point of view of galactic disc formation, the question of the in situ vs. accretion contribution is a key topic as well. In particular, the thick- or thin-disc bimodality has been offered to be explained by either accretion processes such as minor mergers (e.g. Villalobos \& Helmi 2008) or gas infall (Chiappini et al. 1997), or by poor internal secular processes such as radial migration (e.g. Schönrich \& Binney 2009). In this sense, the apparent membership of many metal-rich GCs to the galactic thick disc should once again be linked to these disc formation mechanisms.

In this work, I address the question of the in situ vs. accretion GC formation by focussing on the split of the AMR: are there signs of accretion in the chemical properties of the clusters, and what is their link with the AMR? How do other properties such as the cluster total luminosity vary in the different features of the AMR? What is the transition between the halo and the disc chemical properties? It is important to note that the complex formation and disruption mechanisms analysed through theory and simulations (for an extensive review, see Forbes et al. 2018) hinder the interpretation of present-day GC properties.

With these caveats in mind, literature data of GCs ages, chemical abundances, and structural parameters are studied here in the perspective of the bifurcated age-metallicity relation. In Sect. 2 the observational data are presented. Section 3 compares the GC age-metallicity relation with that of local disc stars. Section 4 explores the $\alpha$-element abundances of GCs in relation with the AMR properties. Section 5 presents an analysis of the absolute magnitude distribution of the clusters. The conclusions are presented in Sect. 6.

\section{Observational data of GCs and disc stars}

This work is based on literature data for the GC analysis and on data from the AMBRE Project (e.g. de Laverny et al. 2012) for the galactic disc comparison sample.

Table 1 presents the complete list of parameters and chemical abundances for the GCs considered in this work. In particular, the following compilations have been used:

- Age estimates have been taken from the homogeneous sample of VandenBerg et al. (2013), completed by Leaman et al. (2013) using the same procedure.

- The $[\mathrm{Fe} / \mathrm{H}]$ abundance corresponds to the Carretta et al. (2009a) scale.

- The total luminosity has been taken from the most recent version of the Harris catalogue (Harris 1996).

- The mean abundance of $\alpha$-elements with respect to iron, in particular $[\mathrm{Mg} / \mathrm{Fe}],[\mathrm{Si} / \mathrm{Fe}],[\mathrm{Ca} / \mathrm{Fe}]$, and $[\mathrm{Ti} / \mathrm{Fe}]$, have been taken where possible from Carretta et al. (2009b) and other papers of the same group (50\% of the sample), and otherwise, from the most recent estimate from the literature (cf. Table 1, Col. 7) ${ }^{1}$.

For the local disc comparison sample, the stars included in the AMBRE Project data have been considered. In particular, the AMBRE:HARPS sample of De Pascale et al. (2014)

\footnotetext{
1 Absolute errors in the abundances for this heterogeneous sample from the literature can be of the order of 0.1 dex.
}

was used. The corresponding $[\mathrm{Fe} / \mathrm{H}]$ and $[\mathrm{Mg} / \mathrm{Fe}]$ are those of Mikolaitis et al. $(2017)^{2}$, while the ages are taken from Hayden et al. (2017) and were determined using Gaia DR1 parallaxes (Lindegren et al. 2016). It is worth noting that possible systematic differences between field stars and GCs could be present in the chemical abundances and the ages because of the different assumptions made in their analysis by different groups.

\section{Age-metallicity relation}

Figure 1 shows the age-metallicity relation found by Leaman et al. (2013), colour-coded following a three-group classification: old metal-poor clusters (black), clusters populating the metal-rich branch of the AMR split (red), and clusters populating the metal-poor branch of the AMR (blue). This threegroups classification is based on the following considerations: i) isolate the metal-rich branch including its oldest clusters; ii) identify the metal-poor branch clusters that are younger than the spread of the age plateau (ranging from 13 to $12 \mathrm{Gyr}$ ), and iii) define a separate class for the oldest metal-poor clusters in the AMR plateau. This arbitrary classification is established for analysis purposes. It allows me to generally compare the properties of the clusters in the different features of the AMR (but see also Kruijssen et al. 2018, for a physically motivated separation by accreted galaxy mass in this parameter space). In addition, as shown in the following sections, the conclusions of the analysis are not influenced by slight modifications of the three groups.

As noted in the Introduction, the metal-rich (red) group is generally considered to be formed in situ and to be associated with the galactic disc. The young metal-poor (blue) group and at least a fraction of the old metal-poor (black) group are assumed to have an accretion origin. This classification into three groups is used in the following sections to compare the chemodynamical and structural parameters of the clusters in these groups and with respect to the local disc field stars.

First of all, Fig. 2 again shows the Leaman et al. (2013) AMR (black points) together with the local thick disc (light blue points) and the local thin disc AMRs (green points) from the AMBRE sample. For comparison purposes, the oldest age limit of the field sample was corrected to be in agreement with the GC one (using a constant shift of $1.5 \mathrm{Gyr}$ that can be explained by modelling-dependent age biases).

On one hand, Fig. 2 shows that the metal-rich branch of the GC AMR bifurcation partially overlaps the locus of thick discs. This suggests that these GCs might have formed in situ during the first epochs of disc formation, in agreement with the Leaman et al. (2013) suggestion of a disc classification of those objects. On the other hand, the metal-poor branch clusters are, as expected, much more metal poor than the thin-disc clusters, with only two clusters (Pal 12 and Ter 7) overlapping the locus of the thin disc AMR.

To better understand if a link can be established between the two GC branches and the disc bimodality, the homogeneous sample of 67 halo stars investigated by Schuster et al. (2012) was included in the plot as orange points. After Nissen \& Schuster (2010) revealed the existence of low- $\alpha$ halo stars, a series of papers of the same group have explored their physical properties in detail, comparing them to canonical high- $\alpha$ halo stars (see also the recent work of Hayes et al. (2018). This confirmed

2 The errors in the abundances are around 0.06 dex (Mikolaitis et al. 2017, Table 4, lower panel). 
Table 1. Adopted parameters for the GCs.

\begin{tabular}{|c|c|c|c|c|c|c|c|c|}
\hline Cluster & {$[\mathrm{Fe} / \mathrm{H}]$} & Age & {$[\mathrm{SiCa} / \mathrm{Fe}]$} & {$[\alpha / \mathrm{Fe}]$} & {$[\mathrm{Mg} / \mathrm{Fe}]$} & $\mathrm{nb}$ & Ref. $\alpha$-abundance & $M_{V}$ \\
\hline NGC 104 & -0.76 & $11.75 \pm 0.25$ & 0.357 & 0.414 & 0.52 & 11 & Carretta et al. (2009b) & -9.00 \\
\hline NGC 288 & -1.32 & $11.50 \pm 0.38$ & 0.391 & 0.379 & 0.45 & 10 & Carretta et al. (2009b) & -6.74 \\
\hline NGC 362 & -1.30 & $10.75 \pm 0.25$ & 0.290 & 0.270 & 0.33 & 92 & Carretta et al. (2013) & -8.41 \\
\hline NGC 1261 & -1.27 & $10.75 \pm 0.25$ & 0.125 & 0.150 & 0.20 & 3 & Filler et al. (2012) & -7.81 \\
\hline NGC 1851 & -1.18 & $11.00 \pm 0.25$ & 0.355 & 0.308 & 0.37 & 119 & Carretta et al. (2011) & -8.33 \\
\hline NGC 2808 & -1.18 & $11.00 \pm 0.38$ & 0.310 & 0.268 & 0.20 & 12 & Carretta et al. (2009b) & -9.39 \\
\hline NGC 3201 & -1.51 & $11.50 \pm 0.38$ & 0.298 & 0.249 & 0.34 & 13 & Carretta et al. (2009b) & -7.46 \\
\hline NGC 4147 & -1.78 & $12.25 \pm 0.25$ & 0.43 & 0.390 & 0.42 & 18 & Villanova et al. (2016) & -6.16 \\
\hline NGC 4590 & -2.27 & $12.00 \pm 0.25$ & 0.331 & 0.282 & 0.35 & 13 & Carretta et al. (2009b) & -7.35 \\
\hline NGC 4833 & -1.89 & $12.50 \pm 0.50$ & 0.405 & 0.333 & 0.37 & 78 & Carretta et al. (2014a) & -8.16 \\
\hline NGC 5024 & -2.06 & $12.25 \pm 0.25$ & 0.350 & 0.300 & 0.33 & 16 & Mészáros et al. (2015) & -8.70 \\
\hline NGC 5053 & -2.30 & $12.25 \pm 0.38$ & 0.385 & 0.385 & & 1 & Sbordone et al. (2015) & -6.72 \\
\hline NGC 5272 & -1.50 & $11.75 \pm 0.25$ & 0.340 & 0.382 & 0.61 & 33 & Carretta et al. (2009b) & -8.93 \\
\hline NGC 5286 & -1.70 & $12.50 \pm 0.38$ & 0.36 & 0.407 & 0.55 & 62 & Marino et al. (2015) & -8.61 \\
\hline NGC 5466 & -2.31 & $12.50 \pm 0.25$ & 0.275 & 0.238 & 0.277 & 3 & Lamb et al. (2015) & -6.96 \\
\hline NGC 5904 & -1.33 & $11.50 \pm 0.25$ & 0.340 & 0.316 & 0.41 & 14 & Carretta et al. (2009b) & -8.81 \\
\hline NGC 5927 & -0.29 & $10.75 \pm 0.38$ & 0.090 & 0.128 & 0.230 & 56 & Recio-Blanco et al. (2017) & -7.80 \\
\hline NGC 5986 & -1.63 & $12.25 \pm 0.75$ & 0.300 & & 0.284 & 25 & Johnson et al. (2017) & -8.44 \\
\hline NGC 6101 & -1.98 & $12.25 \pm 0.50$ & & & & & & -6.91 \\
\hline NGC 6121 & -1.18 & $11.50 \pm 0.38$ & 0.470 & 0.439 & 0.55 & 14 & Carretta et al. (2009b) & -7.20 \\
\hline NGC 6144 & -1.82 & $12.75 \pm 0.50$ & & & & & & -6.75 \\
\hline NGC 6171 & -1.03 & $12.00 \pm 0.75$ & 0.470 & 0.429 & 0.51 & 5 & Carretta et al. (2009b) & -7.13 \\
\hline NGC 6205 & -1.58 & $12.00 \pm 0.38$ & 0.420 & 0.378 & 0.44 & 53 & Carretta et al. (2009b) & -8.70 \\
\hline NGC 6218 & -1.33 & $13.00 \pm 0.50$ & 0.387 & 0.389 & 0.52 & 11 & Carretta et al. (2009b) & -7.32 \\
\hline NGC 6254 & -1.57 & $11.75 \pm 0.38$ & 0.312 & 0.320 & 0.49 & 14 & Carretta et al. (2009b) & -7.48 \\
\hline NGC 6304 & -0.37 & $11.25 \pm 0.38$ & & & & & & -7.32 \\
\hline NGC 6341 & -2.35 & $12.75 \pm 0.25$ & 0.275 & 0.194 & 0.13 & 47 & Mészáros et al. (2015) & -8.20 \\
\hline NGC 6352 & -0.62 & $10.75 \pm 0.38$ & 0.165 & 0.235 & 0.47 & 9 & Feltzing et al. (2009) & -6.48 \\
\hline NGC 6362 & -1.07 & $12.50 \pm 0.25$ & 0.29 & 0.33 & 0.36 & 2 & Gratton (1987) & -6.94 \\
\hline NGC 6366 & -0.59 & $11.00 \pm 0.50$ & 0.275 & 0.290 & 0.29 & 5 & Johnson et al. (2016) & -5.77 \\
\hline NGC 6397 & -1.99 & $13.00 \pm 0.25$ & 0.309 & 0.312 & 0.46 & 13 & Carretta et al. (2009b) & -6.63 \\
\hline NGC 6426 & -2.15 & $12.25 \pm 0.25$ & 0.37 & 0.345 & 0.44 & 4 & Hanke et al. (2017) & -6.69 \\
\hline NGC 6496 & -0.46 & $10.75 \pm 0.38$ & & & & & & -7.23 \\
\hline NGC 6535 & -1.79 & $12.75 \pm 0.50$ & 0.365 & 0.348 & 0.478 & 30 & Bragaglia et al. (2017) & -4.75 \\
\hline NGC 6541 & -1.82 & $12.50 \pm 0.50$ & 0.436 & 0.377 & 0.35 & 3 & Lee \& Carney (2002) & -8.37 \\
\hline NGC 6584 & -1.50 & $11.75 \pm 0.25$ & & & & & & -7.68 \\
\hline NGC 6624 & -0.42 & $11.25 \pm 0.50$ & & & & & & -7.49 \\
\hline NGC 6637 & -0.59 & $11.00 \pm 0.38$ & & & & & & -7.64 \\
\hline NGC 6652 & -0.76 & $11.25 \pm 0.25$ & & & & & & -6.68 \\
\hline NGC 6656 & -1.70 & $12.50 \pm 0.50$ & 0.37 & 0.34 & 0.39 & 35 & Marino et al. (2011) & -8.50 \\
\hline NGC 6681 & -1.62 & $12.75 \pm 0.38$ & 0.43 & 0.41 & 0.52 & 9 & O’Malley et al. (2017) & -7.11 \\
\hline NGC 6715 & -1.44 & $11.75 \pm 0.50$ & 0.34 & 0.285 & 0.28 & 76 & Carretta et al. (2010a) & -10.01 \\
\hline NGC 6717 & -1.26 & $12.50 \pm 0.50$ & & & & & & -5.66 \\
\hline NGC 6723 & -1.10 & $12.50 \pm 0.25$ & 0.33 & 0.28 & 0.23 & 7 & Rojas-Arriagada et al. (2016) & -7.84 \\
\hline NGC 6752 & -1.55 & $12.50 \pm 0.25$ & 0.386 & 0.366 & 0.500 & 14 & Carretta et al. (2009b) & -7.73 \\
\hline NGC 6779 & -2.00 & $12.75 \pm 0.50$ & & & -0.10 & 1 & Khamidullina et al. (2014) & -7.38 \\
\hline NGC 6791 & 0.29 & $8.3 \pm 0.30$ & 0.010 & 0.092 & 0.124 & 32 & Linden et al. (2017) & \\
\hline NGC 6809 & -1.93 & $13.00 \pm 0.25$ & 0.366 & 0.323 & 0.470 & 14 & Carretta et al. (2009b) & -7.55 \\
\hline NGC 6838 & -0.82 & $11.00 \pm 0.38$ & 0.344 & 0.398 & 0.490 & 12 & Carretta et al. (2009b) & -5.60 \\
\hline NGC 6981 & -1.48 & $11.50 \pm 0.25$ & & & & & & -7.04 \\
\hline NGC 7006 & -1.46 & $11.25 \pm 0.25$ & & 0.350 & & 105 & (2008) & -7.68 \\
\hline NGC 7078 & -2.33 & $12.75 \pm 0.25$ & 0.290 & 0.303 & 0.450 & 13 & Carretta et al. (2009b) & -9.17 \\
\hline NGC 7089 & -1.66 & $11.75 \pm 0.25$ & 0.135 & 0.185 & 0.410 & 94 & Recio-Blanco et al. (2017) & -9.02 \\
\hline NGC 7099 & -2.33 & $13.00 \pm 0.25$ & 0.309 & 0.331 & 0.51 & 10 & Carretta et al. (2009b) & -7.43 \\
\hline Arp 2 & -1.74 & $12.00 \pm 0.38$ & 0.32 & 0.31 & 0.38 & 2 & Mottini et al. (2008) & -5.29 \\
\hline Pal 12 & -0.81 & $9.0 \pm 0.38$ & -0.035 & -0.045 & 0.08 & 4 & Cohen (2004) & -4.48 \\
\hline Ter 8 & -2.34 & $13.00 \pm 0.38$ & 0.220 & 0.240 & 0.47 & 7 & Carretta et al. (2014b) & -5.05 \\
\hline Ter 7 & -0.45 & $7.75 \pm 0.50$ & 0.035 & -0.020 & -0.11 & 5 & Sbordone et al. (2005) & -5.05 \\
\hline IC 4499 & -1.62 & $11.25 \pm 0.25$ & & & & & & -7.33 \\
\hline Rupr 106 & -1.78 & $10.75 \pm 0.25$ & -0.020 & 0.000 & -0.02 & 9 & Villanova et al. (2013) & -6.35 \\
\hline Pyxis & -1.20 & $10.50 \pm 0.25$ & & & & & & -5.75 \\
\hline
\end{tabular}

Notes. Metallicity (Col. 2, in dex), age (Col. 3, in Gyr), $\alpha$-element chemical abundances in dex (mean of [Si/Fe] and $[\mathrm{Ca} / \mathrm{Fe}]$ in Col. 4; mean of $[\mathrm{Si} / \mathrm{Fe}],[\mathrm{Ca} / \mathrm{Fe}],[\mathrm{Ti} / \mathrm{Fe}]$, and $[\mathrm{Mg} / \mathrm{Fe}]$ in $\mathrm{Col} .5 ;[\mathrm{Mg} / \mathrm{H}]$ in Col. 6), number of observed stars (Col. 7) and their corresponding reference $(\mathrm{Col} .8)$, and absolute magnitude (Col. 9). 


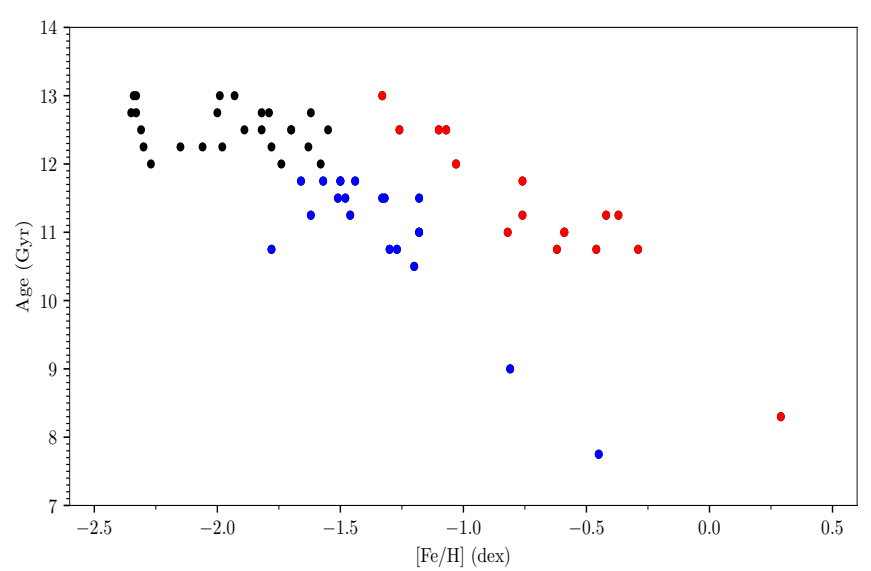

Fig. 1. Leaman et al. (2013) age-metallicity relation, colour-coded to define three groups of clusters: old metal-poor clusters (black), metalrich branch clusters (red), and metal-poor branch (blue) clusters.

the existence of two chemically distinct halo populations in the field halo stars. In particular, Schuster et al. (2012) investigated the mean ages of a sample of high- $\alpha$ and low- $\alpha$ stars as a function of the metallicity (cf. their Table 2). Figure 2 presents these mean values for the high- $\alpha$ stars as orange squares and for the low- $\alpha$ ones as orange circles (the same shift of $1.5 \mathrm{Gyr}$ as was applied to the disc field sample was used). The error bar corresponds to the reported standard deviation at each metallicity bin. The Schuster et al. (2012) stars again present a bimodality. Low $\alpha$ halo objects seem to approximately lie along the metal-poor GC branch and certainly below the metal-rich branch and the thick-disc locus, suggesting a connection with the thin-disc locus at higher metallicities. Nevertheless, the lack of objects in the younger part of the AMR bimodality between -1.2 and -0.8 dex prevents me from robustly concluding about the connection between the metal-poor branch GCs and thindisc GCs. The high- $\alpha$ halo stars, as already known, more or less overlap the thick-disc sequence and the GC metal-rich sequence.

Generally speaking, the GC AMR bimodality seems to have some overlap with the Galactic disc AMR bimodality, especially during the thick-disc formation phase. However, the inhomogeneity of the plot, which includes three different sets of data (clusters, disc, and field halo stars), each of it with its biases in age and metallicity, prevents a conclusion on the relations between them. Despite this, Fig. 2 shows that a bimodality of the AMR is present in the three galactic populations. A more homogeneous data set that includes clusters, field halo stars, and disc stars needs to be analysed to clearly conclude on the substructure of the galactic AMR.

Finally, it is also important to point out that substructures in the age-metallicity plane, such as the above discussed bimodalities, can be created by different evolutionary processes that differently different structural galactic components independently. For instance, accretion from dwarf galaxies in the halo or the disc, gas infall or radial outflows in the disc, radially dependent star formation rates, etc. might all be responsible for an AMR split in a way that the overlap of the clusters and disc features would only be result of a degeneracy in the age-metallicity domain and not the expression of a common evolutionary path. Despite this caveat, the comparative analysis of different galactic components is extremely useful to allow a general vision of the Milky Way evolution and of the complex relations between its different stellar populations.

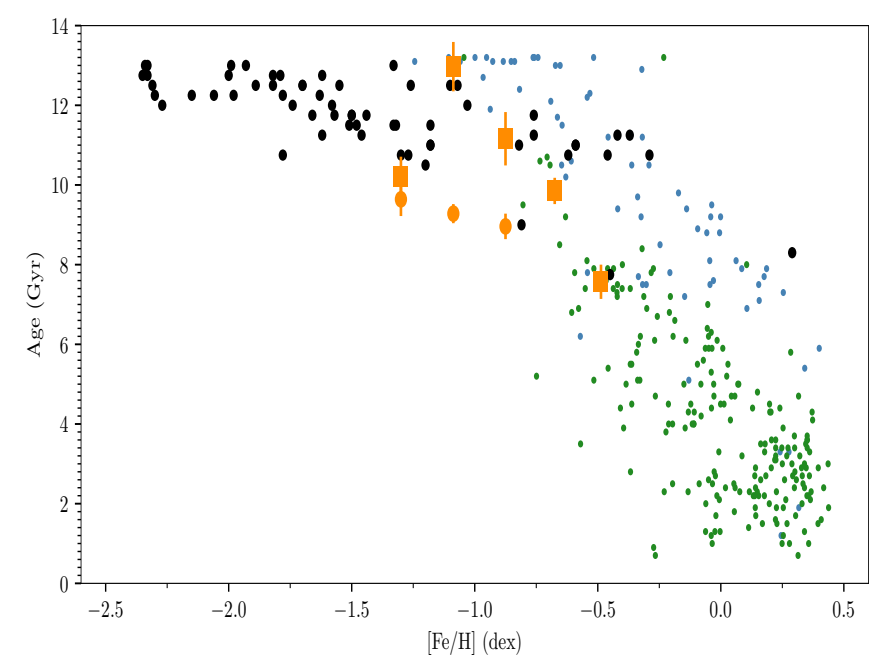

Fig. 2. Leaman et al. (2013) AMR (black points) together with the local thick disc AMR (light blue points) and the local thin disc AMR (green points) from Hayden et al. (2017) and the mean values for the low- $\alpha$ (orange circles) and high- $\alpha$ (orange squares) halo field stars investigated by Schuster et al. (2012; orange).

\section{4. $\alpha$-element abundances and the GC AMR bifurcation}

In this section the mean $\alpha$-element abundance of GCs is examined in relation with the AMR bifurcation. It is well known that the $[\alpha / \mathrm{Fe}]$ chemical abundance ratio is an important indicator of the chemical evolution of a system. In particular, and according to the time-delay model (Tinsley 1979; Matteucci \& Greggio 1986), the initially enhanced $\alpha$-abundance levels with respect to iron start to strongly decline with $[\mathrm{Fe} / \mathrm{H}]$ after the supernovae Ia explosion rate reaches maximum.

On one hand, this produces a knee in the $[\alpha / \mathrm{Fe}]$ vs. $[\mathrm{Fe} / \mathrm{H}]$ trend whose location provides constraints on the star formation rate during the early star formation of a system (e.g. de Boer et al. 2014). As a consequence, the knee location also depends on the system total mass: the less massive the system, the lower the $[\mathrm{Fe} / \mathrm{H}]$ value of the $[\alpha / \mathrm{Fe}]$ turnover. Observations of dwarf galaxies of different masses have confirmed this dependence. A low-mass galaxy like Carina shows a very metal-poor knee $([\mathrm{Fe} / \mathrm{H}]=-2.7 \pm 0.3 \mathrm{dex})$, while the more massive Sculptor presents a slightly more metal-rich knee $([\mathrm{Fe} / \mathrm{H}]=-1.9 \pm 0.1 \mathrm{dex}, \mathrm{McC}$ Connachie 2012). Finally, the knee of higher mass galaxies, like Sagittarius can reach metallicities as high as $[\mathrm{Fe} / \mathrm{H}]=-1.27 \pm 0.05 \mathrm{dex}$ (de Boer et al. 2014). In addition, the Milky Way galaxy has a knee in the range -1.0 dex to -0.5 dex, depending on the authors (de Boer et al. 2014; Rojas-Arriagada et al. 2017)

On the other hand, the initial mass function (IMF) of the system influences the primordial $[\alpha / \mathrm{Fe}]$ and therefore the $[\alpha / \mathrm{Fe}]$ abundance of the low-metallicity plateau. Finally, the $[\alpha / \mathrm{Fe}]$ is a fairly good age indicator for $[\mathrm{Fe} / \mathrm{H}]$ values higher than the knee value (e.g. Haywood et al. 2013; Hayden et al. 2017).

Figure 3 presents $[\mathrm{Mg} / \mathrm{Fe}]$ as a function of $[\mathrm{Fe} / \mathrm{H}]$ for $\mathrm{i}$ ) the Leaman et al. (2013) GCs with available chemical information, using the colour code of Fig. 1, ii) the AMBRE thickand thin-disc comparison sample, and iii) the low- $\alpha$ and high- $\alpha$ halo field stars investigated by Schuster et al. (2012). For clarity, the same colour code as in Fig. 2 is used. Clearly, the GCs show a bimodal behaviour for metallicities higher than about 


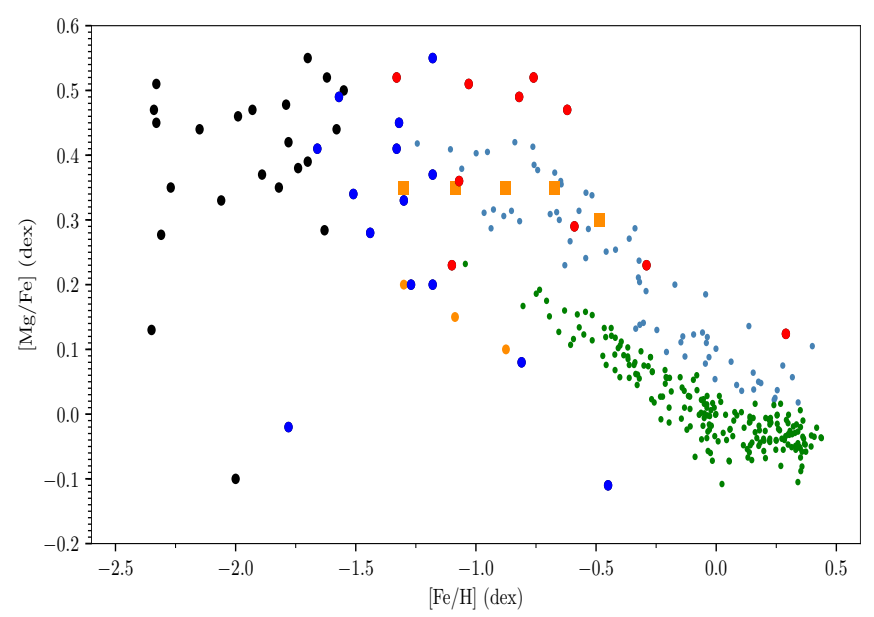

Fig. 3. $[\mathrm{Mg} / \mathrm{Fe}]$ abundance as a function of $[\mathrm{Fe} / \mathrm{H}]$ for the Leaman et al. (2013) GCs, the disc comparison sample, and the Schuster et al. (2012) halo field stars. The colour codes are those of Figs. 1 and 2 for the clusters, the disc, and the halo field stars.

-1.5 dex, although a large scatter is present. Moreover, this bifurcation separates the two cluster populations that were identified in the AMR split: clusters in the metal-rich branch (red points in Figs. 1 and 3) seem to have higher $[\mathrm{Mg} / \mathrm{Fe}]$ values than clusters in the metal-poor branch (blue points in Figs. 1 and 3). This would be in agreement with a possible accreted origin of the metal-poor young clusters, as suggested by Leaman et al. (2013). In addition, when the two disc sequences are considered, the thick-disc sequence presents $[\mathrm{Mg} / \mathrm{Fe}]$ abundances that are compatible with those of the metal-rich branch clusters, again in agreement with a disc origin for these clusters. The thin disc, for its part, shows higher $[\mathrm{Mg} / \mathrm{Fe}]$ abundances than the youngest GCs in the metal-poor branch, although both sequences seem to join at around $[\mathrm{Fe} / \mathrm{H}] \sim-1.0$ dex. Finally, the halo field low- $\alpha$ stars occupy the same locus as the more metal-rich clusters of the metal-poor branch, while the $[\mathrm{Mg} / \mathrm{Fe}]$ abundances of high- $\alpha$ halo field stars are more similar to those of the thick disc and the metal-rich branch clusters (declining for metallicities higher than about $-0.75 \mathrm{dex}$ ). As a consequence, the three studied populations (GCs, disc, and halo field stars) and their sub-classes (metal-rich and metal-poor branch clusters, thick and thin disc, and high- and low- $\alpha$ halo stars) show similar overlaps in the AMR and in the $[\mathrm{Mg} / \mathrm{Fe}]$ vs. $[\mathrm{Fe} / \mathrm{H}]$ planes. It has to be stressed, nevertheless, that the disc, the $\mathrm{GC}$, and the halo sequences are plotted together mainly for a general comparison, as data biases between the two data sets prevent a robust conclusion on their links. In addition, the origin of the Schuster et al. (2012) halo stars is under debate, so that their abundance patterns alone do not provide evidence whether the halo and its GCs formed in situ or ex situ.

Furthermore, only the mean chemical abundances for each cluster are taken into account in Fig. 3, neglecting the wellknown internal light element abundance scatter. GC star formation is currently believed to be bimodal, with a second generation of stars born from the ejecta of the primeval generation (e.g. Carretta et al. 2009b). There is evidence that the $\mathrm{Mg}$-Al cycle is active in cluster polluters, causing the so-called star-to-star anti-correlation between the $\mathrm{Mg}$ and the $\mathrm{Al}$ abundances. In this sense, part of the scatter observed in the GC data in Fig. 3 could come from this internal spread in the $\mathrm{Mg}$ abundances. To solve this problem, Fig. 4 shows the $[\alpha / \mathrm{Fe}]$ vs. $[\mathrm{Fe} / \mathrm{H}]$ of the Leaman et al. (2013) GCs, considering four different $\alpha$-elements

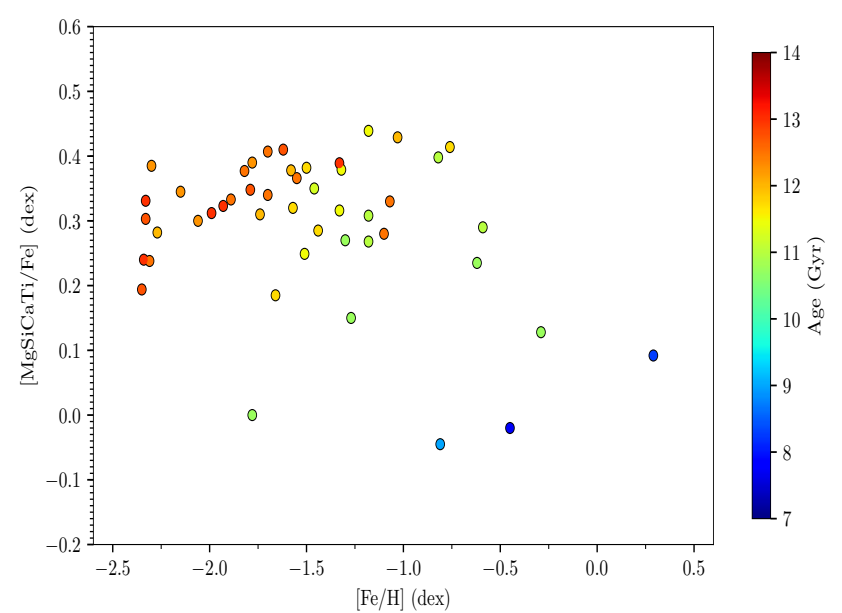

Fig. 4. Mean $[\alpha / \mathrm{Fe}]$ abundance vs. metallicity for the Leaman et al. (2013) GCs, considering four different $\alpha$-elements ( $\mathrm{Mg}, \mathrm{Si}, \mathrm{Ca}$, and Ti). The points are colour-coded by cluster age.

( $\mathrm{Mg}, \mathrm{Si}, \mathrm{Ca}$, and $\mathrm{Ti}$ ). The points are colour-coded by their age. Clearly, the scatter in the more metal-poor clusters is reduced with respect to Fig. 3. In addition, the bifurcation of the abundances at about $[\mathrm{Fe} / \mathrm{H}] \sim-1.5 \mathrm{dex}$, although still present, seems to be less clear, with several metal-poor branch clusters having high $[\alpha / \mathrm{Fe}]$, compatible with those of the older metal-poor population.

Finally, Fig. 5 shows the mean $[\alpha / \mathrm{Fe}]$ abundances considering only $\mathrm{Si}$ and $\mathrm{Ca}$. On one hand, this allows avoiding the scatter due to the $\mathrm{Mg}$ - $\mathrm{Al}$ anti-correlation, and on the other hand, it excludes Ti, which is not a pure $\alpha$-element and is also produced in great abundance by Type Ia supernovae. The Si and Ca mean abundance clearly presents a less strongly scattered high- $\alpha$ sequence of clusters from $-2.5 \mathrm{dex}$ to $-1.0 \mathrm{dex}$. The mean value of the $[\mathrm{SiCa} / \mathrm{Fe}]$ of this sequence is $0.35 \mathrm{dex}$, with only 0.05 dex of standard deviation. This observational scatter is perfectly within the errors of the abundance estimate, especially for an inhomogeneous sample taken from the literature. As a way of comparison, the dispersion for the same clusters in the range $-2.5 \mathrm{dex} \leq[\mathrm{Fe} / \mathrm{H}] \leq-1.0 \mathrm{dex}$ is $0.11 \mathrm{dex}$ in $[\mathrm{Mg} / \mathrm{Fe}]$ and $[\alpha / \mathrm{Fe}]$. Moreover, Fig. 5 shows a knee around $\sim-0.75$ dex, from which a declining sequence of abundances starts that is similar to the thick-disc sequence. Finally, only a few metal-poor clusters present low [SiCa/Fe] abundances (Rupr 106, NGC 7089, NGC 1261, and Pal 12)

To facilitate comparison between the $[\mathrm{SiCa} / \mathrm{Fe}]$ abundance distribution and the AMR cluster groups, Fig. 6 shows the same colour-coding as Fig. 1. Interestingly, the majority of the metalpoor branch clusters present high values of the $[\mathrm{SiCa} / \mathrm{Fe}]$ abundance, equal to (within the error estimates) those of the oldest in situ population and those of the co-eval clusters in the metalrich branch. To facilitate interpretation of this result, Fig. 6 includes the knee positions of the Milky Way (grey vertical band) and those of three different dwarf galaxies following de Boer et al. (2014) and Tolstoy et al. (2009): Sagittarius, Sculptor, and Carina.

Several conclusions can be inferred from Figs. 5 and 6 . First of all, as I described above, the metal-rich branch GCs (in red) follows the Milky Way thick-disc sequence, with a knee at about $[\mathrm{Fe} / \mathrm{H}] \sim-1.0 \mathrm{dex}$. Secondly, the majority of the metal-poor clusters have high $[\mathrm{SiCa} / \mathrm{Fe}]$ abundances with a very low scatter. This indicates that if they have been formed in dwarf galaxies and were subsequently accreted by 


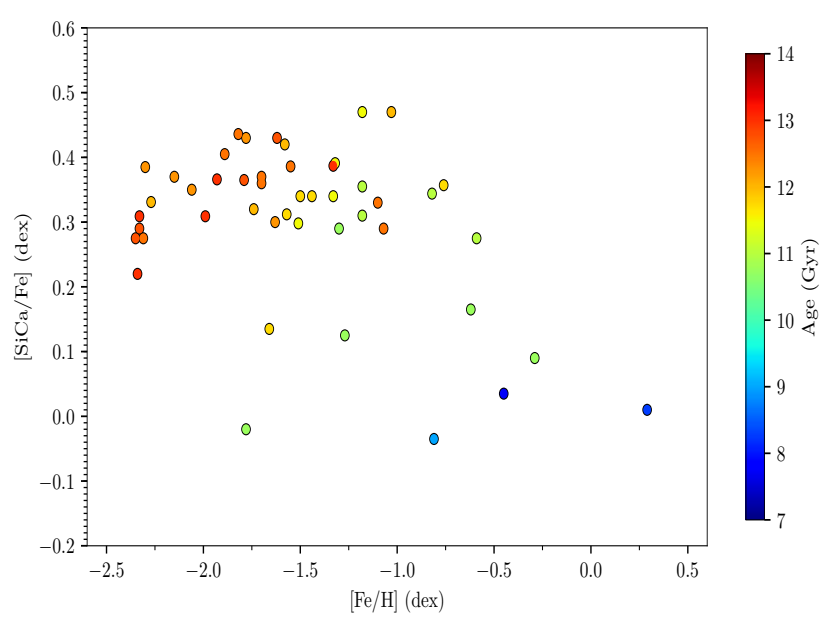

Fig. 5. Mean $[\alpha / \mathrm{Fe}]$ abundances as a function of metallicity for $\mathrm{Si}$ and $\mathrm{Ca}$ alone. The age colour-code is the same as in Fig. 4.

the Milky Way, two possibilities exist: 1) they could all have been formed in massive dwarf galaxies like Sagittarius, which presents a knee in the intermediate-metallicity regime $([\mathrm{Fe} / \mathrm{H}]$ at about -1.2 dex), or 2) they could have been formed in dwarf galaxies with a variety of masses, but have been accreted very early (especially the low-mass dwarfs), before the onset of Type I supernovae causes the decrease in $[\mathrm{SiCa} / \mathrm{Fe}]$ ratio. Conversely, there is also room for an in situ formation of all the metalpoor clusters in the Milky Way halo, which has a metal-rich $[\mathrm{SiCa} / \mathrm{Fe}]$ knee.

Finally, four GCs that are known to be non-monometallic, that is, to have an iron abundance spread (Grebel 2016) (NGC 6656, NGC 6715, NGC 7098, and Terzan 7), are labelled in Fig. 6 with an open black circle. Two of them, Terzan 7 and NGC 7089, clearly present low $[\mathrm{SiCa} / \mathrm{Fe}]$ abundances, which is an unmistakable sign of accretion. In addition, the cluster NGC 6715 (M 54), which is known to be embedded at the centre of the Sagittarius dwarf galaxy, presents a high $[\mathrm{SiCa} / \mathrm{Fe}]$ ratio that is compatible with the high-metallicity knee of the massive Sagittarius.

In conclusion, when $\alpha$-element abundances (not disturbed by the internal scatter of GCs) are considered, the hypothesis of an accretion origin for the metal-poor branch clusters seems to be restricted, with only a few exceptions, to the most massive dwarf galaxies and to early accretion of low-mass dwarfs. On the other hand, and based on the $\alpha$-element abundances alone, their in situ formation cannot be ruled out. Their $\alpha$-abundance patterns are indeed also compatible with the isolated chemical evolution of a high-mass galaxy such as the Milky Way.

\section{Total luminosity distributions}

This section analyses the total visible luminosity of the clusters, taken from the most recent version of the Harris catalogue (Harris 1996). The luminosities are compared with the different groups defined in Sect. 3 from the AMR. This exercise is nevertheless challenging because of the complex physics involved in the formation and disruption of GCs (e.g. Forbes et al. 2018), which hinders the interpretation of the cluster luminosity function. On one hand, the luminosity function of GCs has historically been considered universal and was used as a secondary distance indicator. Recent results have revealed weak dependences on Hubble type, mass, environment, and dynamical history of the host galaxy (e.g. Rejkuba 2012; Harris et al. 2014),

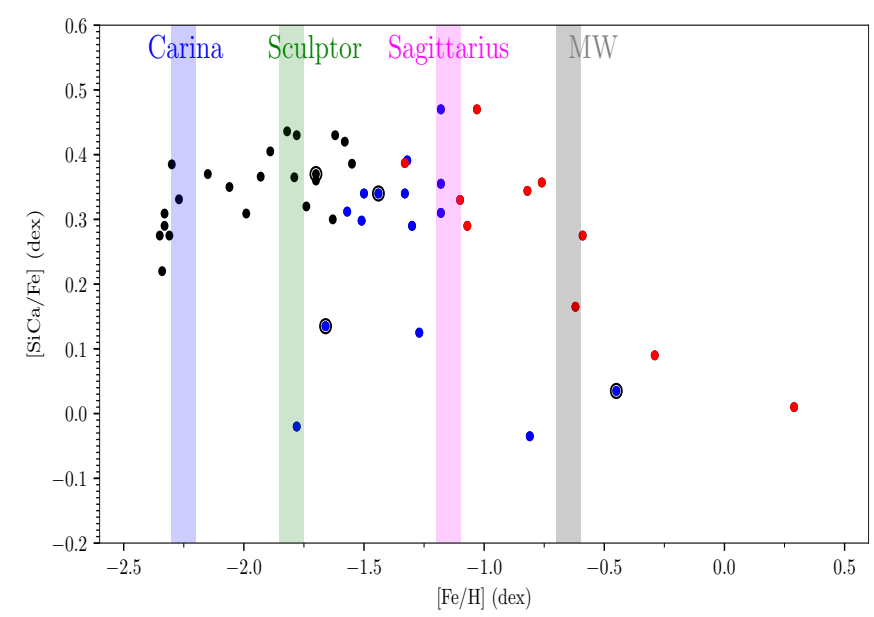

Fig. 6. Same as Fig. 5, but using the colour code of Fig. 1 to distinguish the different groups of clusters in the AMR. The knees of the Milky Way, Sagittarius, Sculptor, and Carina are presented as vertical bands in grey, pink, green, and blue, respectively.

which affect the turnover luminosity (where the luminosity function peaks), only to second order. On the other hand, the luminosity function of Local Group dwarf spheroidals and that of the outer halo Milky Way cluster population are found to contain fainter GCs (van den Bergh 2006; van den Bergh \& Mackey 2004; Carretta et al. 2010b).

With these challenges in mind, the luminosity distribution of the clusters in the Leaman et al. (2013) sample can still be studied to report similarities or differences depending on age and or metallicity. It is important to note that the great majority of bulge clusters are not included in the Leaman et al. (2013) data base. This biases the analysis of the metal-rich population and excludes very high density environments.

First of all, the top panel of Fig. 7 shows the (absorptioncorrected) $M_{V}$ for the old GCs in the AMR plateau (grey histogram) compared to that of the metal-poor (blue histograms) and metal-rich branch clusters (shown in red). As it is difficult to infer the corresponding mass loss of each GC that is lost in the stars that have escaped the cluster, the three histograms in the top panel were smoothed through a kernel density estimation (KDE) technique. To this purpose, two different $\lambda$ covariance factors were used: 0.2 (middle panel of Fig. 7) and 0.5 (lower panel). Finally, a Kolmogorov-Smirnov (K-S) test was performed to compare the three distributions. When the $M_{V}$ distribution of the metal-poor branch young GCs (shown in blue) is compared that of the metal-poor old GCs (shown in grey), the K-S p-value is 0.65 , showing that both distributions are very similar. This result highlights the fact that no particular discontinuity in the typical luminosity of the clusters seems to exist among the metal-poor clusters, even considering an age range of at least 3 Gyr. Conversely, metal-rich branch GCs (in red) are generally less luminous than the others: the total magnitudes of $80 \%$ of the metal-rich branch clusters are fainter than -8.0 , while this number decreases to $50 \%$ and $55 \%$ for the other two subpopulations I analysed (metal-poor branch and old clusters). The K-S p-value that compares the two distributions is 0.33 , which quantifies the lower degree of similarity between the discassociated clusters and the old halo discs. Nevertheless, a final word of caution has to be said about two aspects of this analysis: i) there is not necessarily a mass dependence if a difference in $M v$ is seen because of the difficult interplay between cluster mass and luminosity, which depends on multiple parameters 


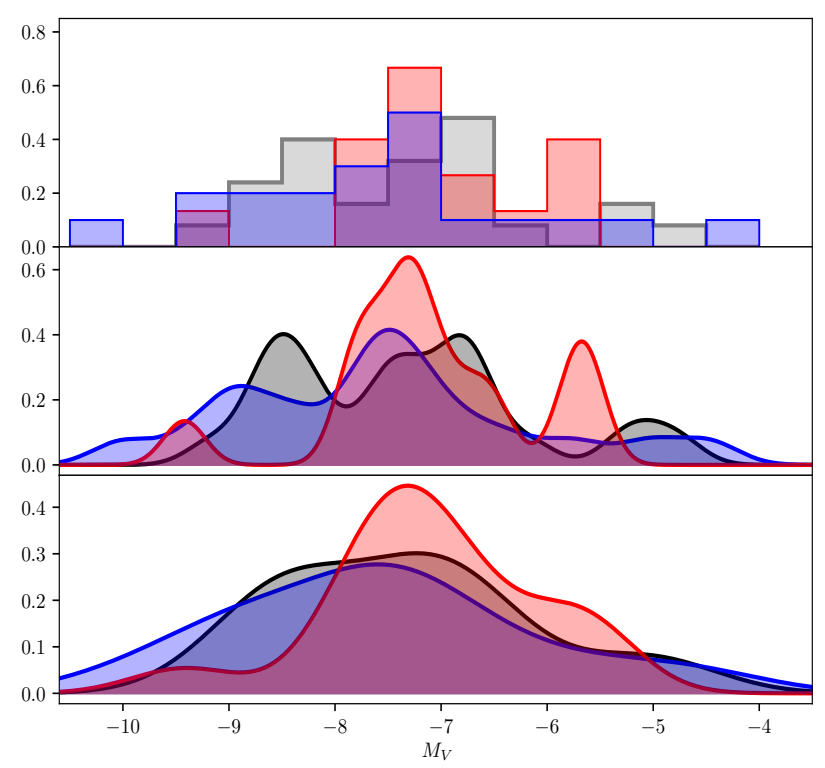

Fig. 7. Top panel: normalised $M_{V}$ distribution of old in situ GCs (grey histogram) compared to that of the metal-poor branch clusters (blue histogram) and that of the metal-rich branch clusters (shown in red). Middle and lower panels: three distributions smoothed through a KDE, with a $\lambda$ covariance factor of 0.2 and 0.5 .

(metallicity, environmental effects, etc.), and ii) the reported $\mathrm{K}-\mathrm{S}$ p-values are estimated in a regime of relatively low-number statistics.

To further examine the nature of the possible luminosity distribution differences between the clusters subpopulations, Fig. 8 explores the chemical dependences on cluster luminosity. The upper panel shows the mean $[\mathrm{SiCa} / \mathrm{Fe}]$ as a function of the total cluster magnitude, using a colour code with age. On one hand, as I highlighted in the previous section, the majority of the clusters appear in a high- $\alpha$ regime $([\mathrm{SiCa} / \mathrm{Fe}] \sim 0.35 \mathrm{dex})$. No particular tendency with $M v$ or age appears for these clusters, which offers no additional constraints about their origin (e.g. massive dwarf galaxies, early accretion of low-mass dwarfs or in situ formation). On the other hand, a less densely populated low- $\alpha$ regime $([\mathrm{SiCa} / \mathrm{Fe}] \leq 0.20 \mathrm{dex}$, with a mixture of metal-rich and metalpoor clusters) is also visible and appears to be clearly separated from the standard high- $\alpha$ regime, especially for the brighter luminosities.

To distinguish the nature of the features seen in the $[\mathrm{SiCa} / \mathrm{Fe}]$ vs. $M v$ plane, the lower panel of Fig. 8 presents the mean $[\mathrm{SiCa} / \mathrm{Fe}]$ as a function of the total cluster magnitude with a colour code showing clusters in the AMR metal-poor branch (blue), the metal-rich branch (red) and the old metal-poor population (grey). First of all, high- $\alpha$ metal-poor branch clusters (which represent the majority of this population and cover the metallicity regime between about $-1.6 \mathrm{dex}$ and $-1.1 \mathrm{dex}$ ) are all brighter than about $M v \sim-7 \mathrm{mag}$. This is not the case for more metal-poor old clusters $([\mathrm{Fe} / \mathrm{H}] \lesssim-1.5 \mathrm{dex}$, grey points), which can be as faint as $-4.5 \mathrm{mag}$. As discussed in the previous section, the high- $\alpha$ metal-poor population could have formed in a mixture of massive and less massive dwarf galaxies, in contrast to the high- $\alpha$ population at normal metallicity level, whose external origin requires higher-mass satellites. The greater luminosity range spanned by the old metal-poor clusters with respect to the high- $\alpha$ clusters in the intermediate metallicity range suggests a higher heterogeneity of formation (and eventually, distruption) environments at lower metallicity.

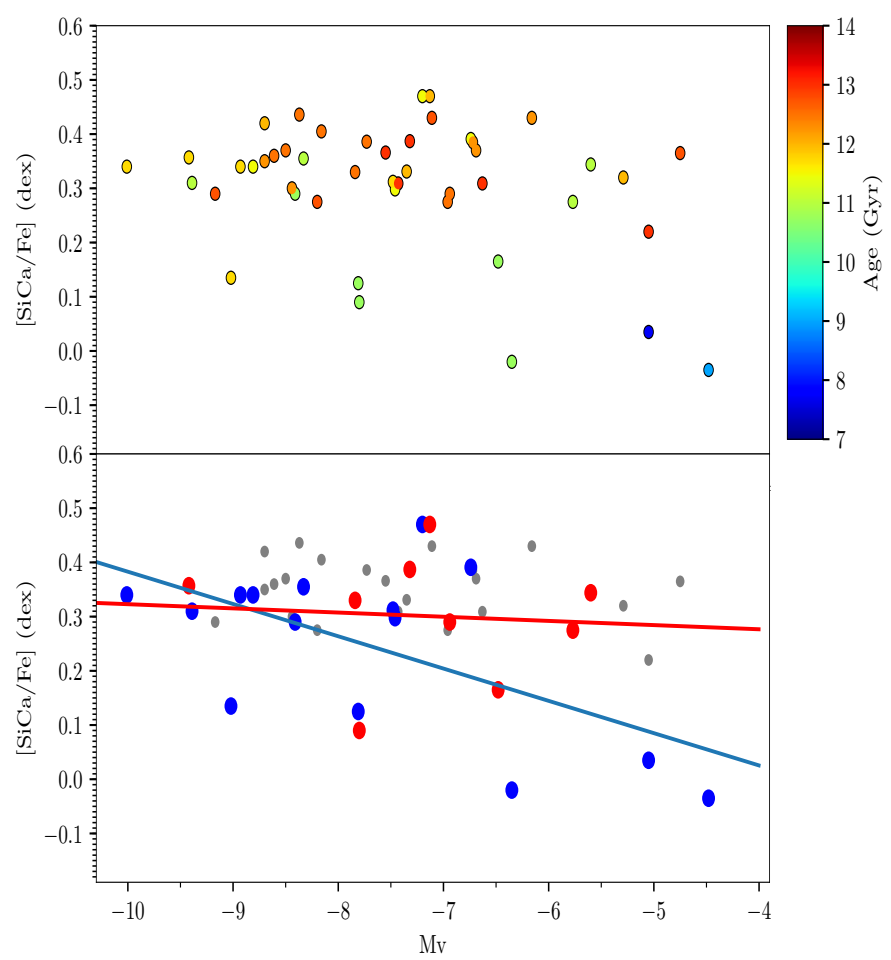

Fig. 8. Top panel: $[\alpha / \mathrm{Fe}]$ abundances as a function of total cluster magnitude for $\mathrm{Si}$ and $\mathrm{Ca}$ alone. Points are colour coded by age. Lower panel: same as the upper panel, but identifying clusters in the AMR metal-poor branch (blue points), the AMR metal-rich branch (red points), and the old metal-poor population (black points). The solid blue and red lines correspond to the linear fit of the metal-poor and the metal-rich branch clusters, respectively. An anti-correlation of the $[\mathrm{SiCa} / \mathrm{Fe}]$ abundance with the total magnitude $(r=-0.59)$ seems to be present only for metalpoor branch clusters.

Secondly, when the few clusters with $[\mathrm{SiCa} / \mathrm{Fe}] \leq 0.20 \mathrm{dex}$ are also considered, metal-poor branch clusters present an anticorrelation ( $r=-0.59$, solid blue line) of $[\mathrm{SiCa} / \mathrm{Fe}]$ with $M v$, while this is not the case for metal-rich branch clusters $(r=$ -0.08 , solid red line). This difference between the two subpopulations is strongly driven by the fact that no faint high- $\alpha$ clusters are observed among the metal-poor branch subpopulation, in contrast to the metal-rich branch population. Although the reasons for such a difference can be multiple and degenerate, and the number of clusters with available data is not very high, I point out that clusters that are separated in the AMR plane seem different in their chemical properties as well when the low-luminosity regime is considered. The environment in which faint metal-poor branch clusters have formed and evolved could have been subject to lower star formation rate or preferential loss of elements from massive supernova type II than the environment that hosts faint metal-rich branch clusters. These two conditions are compatible with the formation of the faint metal-poor branch clusters in dwarf galaxies, and therefore are compatible also with their accreted origin. In addition, the observed anti-correlation of the $[\mathrm{SiCa} / \mathrm{Fe}]$ ratio with $M v$ for metal-poor branch clusters is also puzzling, suggesting that their chemical evolution conditions might be different for brighter clusters (higher star formation rate or more efficient massive supernova feedback) than for fainter clusters. Although the available data do not allow concluding on this, one possibility would be that more massive dwarfs would host more luminous clusters, as has been suggested by van den Bergh (2006). 


\section{Summary and discussion}

Different aspects of the galactic GC populuation are revealed by the analysis of the substructures in the Milky Way GC agemetallicity relation, coupled with chemical abundance information, the study of the total luminosity distribution, and the comparison with field stars data. First of all, the bimodality in the age-metallicity relation, reported by Leaman et al. (2013), seems to overlap in time with the halo field star bimodality (Schuster et al. 2012) and the thick-thin disc bimodality (Hayden et al. 2017), although the biases between the different datasets prevent us from a detailed conclusion on their relative links.

Secondly, when the $\alpha$-element abundances, which are less strongly affected by internal light-element spread of GCs, are considered, a very low observational scatter among the metalpoor clusters is observed. A plateau at $[\mathrm{SiCa} / \mathrm{Fe}] \sim 0.35 \mathrm{dex}$, with a dispersion of only $0.05 \mathrm{dex}$, is observed up to a metallicity of about -0.75 dex. Only a few metal-poor clusters in this metallicity interval (Rupr 106, NGC 7089, NGC 1261 and Pal 12) present low [SiCa/Fe] abundances. Moreover, metal-rich GCs follow the Milky Way thick-disc sequence, with a knee at about $[\mathrm{Fe} / \mathrm{H}] \sim-0.75$ dex. This result places a clear constraint on scenarios of GC formation in the Milky Way. On one hand, if a substantial fraction of galactic GCs has an external origin, they must have formed either in galaxies that were massive enough to ensure high levels of $\alpha$-element abundances even at intermediate metallicity (e.g. the Sagittarius dwarf galaxy), or in lowermass dwarf galaxies that accreted in their early formation phase (before they reached their $[\alpha / \mathrm{Fe}]$ vs. $[\mathrm{Fe} / \mathrm{H}]$ knee). On the other hand, the in situ formation of clusters with high $[\alpha / \mathrm{Fe}]$ values is also a plausible explanation.

Finally, the study of the absolute luminosity distribution in the different features of the AMR reveals that the total luminosity distributions of old metal-poor GCs in the AMR plateau are similar to those of younger clusters of intermediate metallicity. However, when only high- $\alpha$ clusters are considered, old metal-poor clusters seem to span a wider luminosity range than younger clusters of intermediate metallicity. In addition, this lack of faint high- $\alpha$ clusters in the AMR metal-poor branch subpopulation also contradicts what is observed for the metal-rich branch population and reinforces the differences between these two AMR branches. Moreover, metal-rich branch clusters are generally less luminous than metal-poor clusters.

In conclusion, although the interpretation of the differently biased data samples is complex and multi-parametric, the analysis reported here places simple observational constraints on scenarios of GC formation and disruption:

- The duplicity of the Milky Way GC population, illustrated by its bifurcated age-metallicity relation, is confirmed by the combined analysis of the $[\alpha / \mathrm{Fe}]$ abundances and the total luminosity distributions. Some overlap with the disc population in the various bimodalities is observed, although common evolutionary paths for the disc and the GC populations are not guaranteed because of the observational biases and the degeneracy in the effects of different physical evolutionary processes.

- The external origin of metal-poor branch clusters seems reinforced (or at least not excluded) by the $[\alpha / \mathrm{Fe}]$ abundances.

- The greater luminosity range that is spanned by the old metal-poor clusters with respect to the high- $\alpha$ metalintermediate clusters suggests a higher heterogeneity of formation environments at lower metallicity, which might reflect the contribution of low-mass satellite accretion.
- Accretion of high-mass satellites, as a major contribution to the current Milky Way GC system in the metal-poor and intermediate-metallicity regimes, is compatible with the observations.

Generally speaking, the duality of the GC in situ vs. accretion formation scenarios remains only partially unveiled, but the combined analysis of the AMR, the chemical abundances, and the cluster luminosities gives some constraints on the accretion epochs and/or the masses of the accreted objects. Precise dynamical data are of course another crucial piece of the puzzle. The Gaia mission, from its second data release, has already started to open new paths of exploration. In particular, several studies have suggested the accretion of a high-mass satellite to which several GCs are associated, which might have built up the halo inner regions and perturbed the primordial disc (Helmi et al. 2018; Kruijssen et al. 2018; Myeong et al. 2018). Moreover, the agemetallicity relation of halo field stars needs to be studied and placed in relation with the GC AMR in order to confirm a possible, and expected, link between field stars and clusters.

Acknowledgements. I acknowledge financial support from the ANR 14-CE33014-01. I thank all the people involved in the AMBRE project for the use of the AMBRE:HARPS data as a comparison disc sample. I am sincerely grateful to the anonymous referee of this paper for their careful reading of the work and for all the suggestions for improvement. In particular, their help has been crucial for the results in Sect. 5. I warmly thank Patrick de Laverny for his constant support, personal and professional.

\section{References}

Bragaglia, A., Carretta, E., D’Orazi, V., et al. 2017, A\&A, 607, A44 Buonanno, R., Corsi, C. E., Pulone, L., Fusi Pecci, F., \& Bellazzini, M. 1998, A\&A, 333, 505

Carretta, E., Bragaglia, A., Gratton, R., D’Orazi, V., \& Lucatello, S. 2009a, A\&A, 508, 695

Carretta, E., Bragaglia, A., Gratton, R., \& Lucatello, S. 2009b, A\&A, 505, 139

Carretta, E., Bragaglia, A., Gratton, R. G., et al. 2010a, A\&A, 520, A95

Carretta, E., Bragaglia, A., Gratton, R. G., et al. 2010b, A\&A, 516, A55

Carretta, E., Lucatello, S., Gratton, R. G., Bragaglia, A., \& D’Orazi, V. 2011, A\&A, 533, A69

Carretta, E., Bragaglia, A., Gratton, R. G., et al. 2013, A\&A, 557, A138

Carretta, E., Bragaglia, A., Gratton, R. G., et al. 2014a, A\&A, 564, A60

Carretta, E., Bragaglia, A., Gratton, R. G., et al. 2014b, A\&A, 561, A87

Chiappini, C., Matteucci, F., \& Gratton, R. 1997, ApJ, 477, 765

Cohen, J. G. 2004, AJ, 127, 1545

De Angeli, F., Piotto, G., Cassisi, S., et al. 2005, AJ, 130, 116

de Boer, T. J. L., Belokurov, V., Beers, T. C., \& Lee, Y. S. 2014, MNRAS, 443, 658

de Laverny, P., Recio-Blanco, A., Worley, C. C., \& Plez, B. 2012, A\&A, 544, A126

De Pascale, M., Worley, C. C., de Laverny, P., et al. 2014, A\&A, 570, A68

Dotter, A., Sarajedini, A., \& Anderson, J. 2011, ApJ, 738, 74

Feltzing, S., Primas, F., \& Johnson, R. A. 2009, A\&A, 493, 913

Filler, D., Ivans, I. I., \& Simmerer, J. 2012, AAS Meeting Abstracts, 219, 152.02

Forbes, D. A., \& Bridges, T. 2010, MNRAS, 404, 1203

Forbes, D. A., Spitler, L. R., Strader, J., et al. 2011, MNRAS, 413, 2943

Forbes, D. A., Bastian, N., Gieles, M., et al. 2018, Proc. R. Soc. London, Ser. A, 474, 20170616

Gratton, R. G. 1987, A\&A, 179, 181

Grebel, E. K. 2016, in Star Clusters and Black Holes in Galaxies across Cosmic Time, eds. Y. Meiron, S. Li, F.-K. Liu, \& R. Spurzem, IAU Symp., 312, 157

Hanke, M., Koch, A., Hansen, C. J., \& McWilliam, A. 2017, A\&A, 599, A97

Harris, W. E. 1996, AJ, 112, 1487

Harris, W. E. 2009, ApJ, 699, 254

Harris, W. E., Morningstar, W., Gnedin, O. Y., et al. 2014, ApJ, 797, 128

Hayden, M. R., Recio-Blanco, A., de Laverny, P., Mikolaitis, S., \& Worley, C. C. 2017, A\&A, 608, L1

Hayes, C. R., Majewski, S. R., Shetrone, M., et al. 2018, ApJ, 852, 49

Haywood, M., Di Matteo, P., Lehnert, M., Katz, D., \& Gomez, A. 2013, A\&A, 560, A109 
Helmi, A., Babusiaux, C., Koppelman, H. H., et al. 2018, Nature, 563, 85

Johnson, C. I., Caldwell, N., Rich, R. M., Pilachowski, C. A., \& Hsyu, T. 2016, AJ, 152, 21

Johnson, C. I., Caldwell, N., Rich, R. M., et al. 2017, ApJ, 842, 24

Khamidullina, D. A., Sharina, M. E., Shimansky, V. V., \& Davoust, E. 2014, Astrophys. Bull., 69, 409

Kirby, E. N., Guhathakurta, P., \& Sneden, C. 2008, ApJ, 682, 1217

Kruijssen, J. M. D., Pfeffer, J. L., Reina-Campos, M., Crain, R. A., \& Bastian, N. 2018, MNRAS, in press [arXiv:1806.05680]

Lamb, M. P., Venn, K. A., Shetrone, M. D., Sakari, C. M., \& Pritzl, B. J. 2015, MNRAS, 448, 42

Leaman, R., VandenBerg, D. A., \& Mendel, J. T. 2013, MNRAS, 436, 122

Lee, J.-W., \& Carney, B. W. 2002, AJ, 124, 1511

Lindegren, L., Lammers, U., Bastian, U., et al. 2016, A\&A, 595, A4

Linden, S. T., Pryal, M., Hayes, C. R., et al. 2017, ApJ, 842, 49

Marín-Franch, A., Aparicio, A., Piotto, G., et al. 2009, ApJ, 694, 1498

Marino, A. F., Sneden, C., Kraft, R. P., et al. 2011, A\&A, 532, A8

Marino, A. F., Milone, A. P., Karakas, A. I., et al. 2015, MNRAS, 450, 815

Matteucci, F., \& Greggio, L. 1986, A\&A, 154, 279

McConnachie, A. W. 2012, AJ, 144, 4

Mészáros, S., Martell, S. L., Shetrone, M., et al. 2015, AJ, 149, 153

Mikolaitis, Š., de Laverny, P., Recio-Blanco, A., et al. 2017, A\&A, 600 A22

Mottini, M., Wallerstein, G., \& McWilliam, A. 2008, AJ, 136, 614

Myeong, G. C., Evans, N. W., Belokurov, V., Sanders, J. L., \& Koposov, S. E. 2018, ApJ, 863, L28

Nissen, P. E., \& Schuster, W. J. 2010, A\&A, 511, L10
O’Malley, E. M., Knaizev, A., McWilliam, A., \& Chaboyer, B. 2017, ApJ, 846, 23

Pota, V., Forbes, D. A., Romanowsky, A. J., et al. 2013, MNRAS, 428, 389 Recio-Blanco, A., Rojas-Arriagada, A., de Laverny, P., et al. 2017, A\&A, 602, L14

Rejkuba, M. 2012, Ap\&SS, 341, 195

Rojas-Arriagada, A., Zoccali, M., Vásquez, S., et al. 2016, A\&A, 587, A95

Rojas-Arriagada, A., Recio-Blanco, A., de Laverny, P., et al. 2017, A\&A, 601, A140

Rosenberg, A., Saviane, I., Piotto, G., \& Aparicio, A. 1999, AJ, 118, 2306

Salaris, M., \& Weiss, A. 2002, A\&A, 388, 492

Sbordone, L., Bonifacio, P., Marconi, G., Buonanno, R., \& Zaggia, S. 2005, A\&A, 437, 905

Sbordone, L., Monaco, L., Moni Bidin, C., et al. 2015, A\&A, 579, A104

Schönrich, R., \& Binney, J. 2009, MNRAS, 399, 1145

Schuster, W. J., Moreno, E., Nissen, P. E., \& Pichardo, B. 2012, A\&A, 538, A21

Tinsley, B. M. 1979, ApJ, 229, 1046

Tolstoy, E., Hill, V., \& Tosi, M. 2009, ARA\&A, 47, 371

van den Bergh, S. 2006, AJ, 131, 304

van den Bergh, S., \& Mackey, A. D. 2004, MNRAS, 354, 713

VandenBerg, D. A. 2000, ApJS, 129, 315

VandenBerg, D. A., Brogaard, K., Leaman, R., \& Casagrande, L. 2013, ApJ, 775, 134

Villalobos, Á., \& Helmi, A. 2008, MNRAS, 391, 1806

Villanova, S., Geisler, D., Carraro, G., Moni Bidin, C., \& Muñoz, C. 2013, ApJ, 778,186

Villanova, S., Monaco, L., Moni Bidin, C., \& Assmann, P. 2016, MNRAS, 460, 2351 